\title{
Notas taxonômicas em Verbenaceae da Flora do Brasil
}

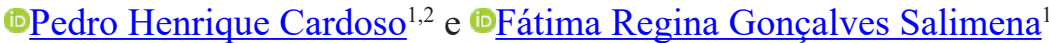

Recebido: 19 janeiro 2019; aceito: 8 outubro 2019

Como citar: Cardoso, P.H. \& Salimena, F.R.G. 2019. Notas taxonômicas em Verbenaceae da Flora do Brasil. Hoehnea 46: e032019. http://dx.doi.org/10.1590/2236-8906-03/2019.

ABSTRACT - (Taxonomic notes in Verbenaceae of the Brazilian Flora). The taxonomic identity of Lantana glaziovii Moldenke and Lippia nana Schauer is revised. These taxa have been accepted as synonyms of Lippia grandiflora Mart. \& Schauer and Lippia pumila Cham., respectively. After a detailed analysis of protologues, herbarium collections, and relevant literature, Lantana glaziovii and Lippia nana are reestablished and accepted here as autonomous species. In addition, a lectotype is designated for Lippia nana.

Keywords: Lantaneae, Lippia grandiflora, Lippia pumila, reestablishment, typification

RESUMO - (Notas taxonômicas em Verbenaceae da Flora do Brasil). A identidade taxonômica de Lantana glaziovii Moldenke e Lippia nana Schauer é revisada. Estes táxons vêm sendo tratados como sinônimos de Lippia grandiflora Mart. \& Schauer e Lippia pumila Cham., respectivamente. Após análise detalhada de protólogos, coleções de herbários e literatura relevante, Lantana glaziovii e Lippia nana são restabelecidas e reconhecidas como espécies autônomas. Além disso, um lectótipo é designado para Lippia nana.

Palavras-chave: Lantaneae, Lippia grandiflora, Lippia pumila, restabelecimento, tipificação

\section{Introdução}

Verbenaceae J.St-Hil compreende cerca de 800 espécies em 34 gêneros, destacando-se Lantana L. e Lippia L. como os mais representativos da tribo Lantaneae, ambos com centro de diversidade na América do Sul (Atkins 2004). A morfologia do fruto é o caráter classicamente utilizado para delimitá-los, de modo que as espécies que apresentam fruto esquizocarpo formado por 2 mericarpos ou drupa com 2 pirenos estão incluídas em Lippia, enquanto as espécies com fruto do tipo drupa 1-pirenado caracterizam Lantana (Silva \& Salimena 2002).

Ao longo do tempo, os gêneros Lantana e Lippia passaram por circunscrições distintas por existirem poucas características diagnósticas para distingui-los (Bentham 1876, Briquet 1895, Junell 1934, Moldenke 1965, Troncoso 1974, Silva \& Salimena 2002). A grande riqueza de espécies e os diversos sinônimos estabelecidos criaram enormes dificuldades taxonômicas infragenéricas e infraespecíficas. Análises filogenéticas recentes indicaram que estes gêneros, conforme formalmente circusncritos, não são monofiléticos, visto que o tipo de fruto representa um caráter homoplástico. Desta forma, tais classificações constituem esquemas artificiais, e no futuro Lippia e Lantana deverão ser admitidos em um único gênero ou fragmentados em vários (Lu-Irving \& Olmstead 2013).

Com o estudo taxonômico da família Verbenaceae para o projeto "Flora do Brasil 2020", em andamento, propõe-se o restabelecimento das espécies Lantana glaziovii Moldenke e Lippia nana Schauer, antes tratadas como sinônimos de Lippia grandiflora Mart. \& Schauer e Lippia pumila Cham., respectivamente (Salimena \& Múlgura 2015). São apresentadas descrições, tipificação, comentários taxonômicos, fotografias e dados sobre distribuição geográfica, hábitat, fenologia e conservação.

\section{Material e métodos}

Este estudo está baseado na revisão acurada dos protólogos, tipos ou imagens de tipos disponíveis

1. Universidade Federal de Juiz de Fora, Instituto de Ciências Biológicas, Campus Universitário, Rua José Lourenço Kelmer, s/n, São Pedro, 36036-900 Juiz de Fora, MG, Brasil

2. Autor para correspondência: pedro.cardoso@ecologia.ufjf.br 
no JSTOR Global Plants, bem como na análise das coleções dos herbários BHCB, BR, CEN, CESJ, ESA, G, HUFU, IBGE, K, MBM, MO, NY, P, RB, SI, SPF, UFG e W (siglas segundo Thiers 2019), visitados e/ou examinados virtualmente. A terminologia morfológica utilizada nas descrições segue Radford et al. (1974), Harris \& Harris (2003) e Gonçalves \& Lorenzi (2007). Dados sobre distribuição, hábitat e fenologia foram obtidos nas etiquetas dos espécimes. O status de conservação das espécies está de acordo com a IUCN (2017), sendo a extensão de ocorrência (EOO) e a área de ocupação (AOO) calculadas utilizando a ferramenta GeoCAT (Bachman et al. 2011).

\section{Resultados e Discussão}

\section{Lantana glaziovii Moldenke, Phytologia 1: 462.} 1940.

Figura 1a-b

Tipo-Brasil. Gorás: On the central plateau of Goyaz, 1894-95, A.F.M. Glaziou 21892 (holótipo G (G00366437); isótipos NY (NY00137646), SI (SI003454), BR (BR0000005504640) [pro parte], $\mathrm{P}$ (P00713621) [pro parte], $\mathrm{P}(\mathrm{P} 00713622)$ [pro parte]).

Subarbustos 3-20 cm alt., monoicos, ramos cilíndricos, densamente hirsutos, tricomas glandulares sésseis ferrugíneos presentes, sistema subterrâneo desenvolvido. Folhas opostas ou 3-verticiladas, sésseis ou curto-pecioladas, pecíolos 0,5-2 mm compr.; lâminas 0,5-1,5 × 0,5-0,7 cm, cartáceas, orbiculares ou ovadas, base cuneada, ápice agudo ou obtuso, margem profundamente crenada, face adaxial hirsuta, face abaxial densamente hirsuta, tricomas glandulares sésseis ferrugíneos presentes. Inflorescências 1-2 $\times 0,6-1,3 \mathrm{~cm}$, hemisféricas, pedúnculo $1,5-4,5 \mathrm{~cm}$ compr., delgado, hirsuto, tricomas glandulares sésseis ferrugíneos presentes, raque não alongada na infrutescência; brácteas ovadas 5-6 mm compr., iguais, dispostas espiraladamente, membranáceas, verdes, densamente hirsutas externamente, tricomas glandulares sésseis ferrugíneos abundantes; cálice ca. 1,5 mm compr., tubuloso, 4-lobulado, lóbulos arredondados, verde, pubescente; corola 7-8 $\mathrm{mm}$ compr., zigomorfa, lilás, fauce amarela, externamente pubescente, tricomas glandulares sésseis ferrugíneos abundantes; ovário ca. 1,5 mm compr., 1-carpelar, 2-locular, lóculos 1-ovulados, estames didínamos, inseridos na metade do tubo. Fruto do tipo drupa 1-pirenado, mesocarpo suculento, esférico, ca. $5 \mathrm{~mm}$ diâm., superfície externa rugosa.
Lantana glaziovii foi descrita por Moldenke (1940), com base nas coleções de Glaziou procedentes do Estado de Goiás. Trata-se de um subarbusto reduzido, apresentando sistema subterrâneo desenvolvido, indumento hirsuto com tricomas glandulares sésseis ferrugíneos, folhas jovens orbiculares e adultas ovadas, pedúnculos delgados, brácteas ovadas e corola lilás com fauce amarela (Silva 1999). Esta espécie foi considerada como sinônimo de Lippia grandiflora por Salimena \& Múlgura (2015) com base no xilopódio desenvolvido, hábito reduzido, fruto com dois mericarpos e floração pós-fogo.

Diversos representantes de Lantana e Lippia vêm sendo erroneamente identificados nas coleções de herbários, com base unicamente na semelhança do hábito reduzido. Por meio de um estudo mais detalhado das características morfológicas das espécies de Lippia para a monografia do gênero no projeto "Flora do Brasil 2020", foi possível verificar a presença de fruto do tipo drupa 1-pirenado para Lantana glaziovii, e dessa forma, reconhecê-la como espécie distinta no gênero Lantana.

As coleções de Glaziou 21892 representadas pelo holótipo depositado no herbário G (G00366437) e pelos isótipos depositados em NY (NY00137646) e SI (SI003454) correspondem de fato somente à Lantana glaziovii. No entanto, os isótipos depositados em BR (BR0000005504640) e P (P00713622 e P00713621) apresentam, na mesma exsicata, espécimes de Lippia horridula (Epling) Salimena, Múlgura \& Harley, sendo que na exsicata depositada em BR eles estão assinalados pela letra "b", e nas exsicatas depositadas em $\mathrm{P}$ indicados pelo número " 1 ".

Desta forma, a sinonímia de Lantana glaziovii sob Lippia grandiflora, fundamentada pela semelhança do hábito e pelo fruto típico de Lippia deu-se na verdade a partir dos frutos de Lippia horridula incluídos nas coleções-tipo de Lantana glaziovii. A sinonimização proposta de Lantana glaziovii em Lippia grandiflora também constituti um erro, pois esta espécie é muito próxima de Lippia horridula e foi identificada como tal pela ausência de folhas adultas, que representa o melhor caráter para identificação e distinção entre estas duas espécies de Lippia. Sendo assim, a confusão taxonômica se estabeleceu pela semelhança entre estes táxons que apresentam nítida floração pós-fogo e, portanto, hábito reduzido, folhas jovens diminutas e corolas vistosas (Salimena \& Múlgura 2015).

Lantana glaziovii pode ser reconhecida por apresentar folhas orbiculares ou ovadas, margem 
profundamente crenada, hirsuta em ambas as faces, tricomas glandulares sésseis ferrugíneos, brácteas dispostas espiraladamente e fruto do tipo drupa 1-pirenado. Estes caracteres permitem distingui-la de Lippia grandiflora por que possui folhas oblongoelípticas, com margem crenada da metade até o ápice, densamente pubescentes em ambas as faces, tricomas glandulares pedicelados alvos e fruto do tipo esquizocarpo formado por dois mericarpos, e de Lippia horridula que apresenta folhas elípticas a estreito-elípticas, com margem grosso-serreada, esparso-estrigosas e brácteas alternas (tabela 1).

Lantana glaziovii está amplamente distribuída no Brasil, ocorrendo em áreas de cerrado nos Estados de São Paulo, Minas Gerais, Goiás, Distrito Federal, Mato Grosso e Tocantins. Já Lippia grandiflora, que apresentava distribuição anterior mascarda em virtude da sinonimização (Salimena \& Múlgura 2015, BFG 2015), é endêmica do cerrado de Minas Gerais, sendo considerada uma espécie rara, com poucos registros
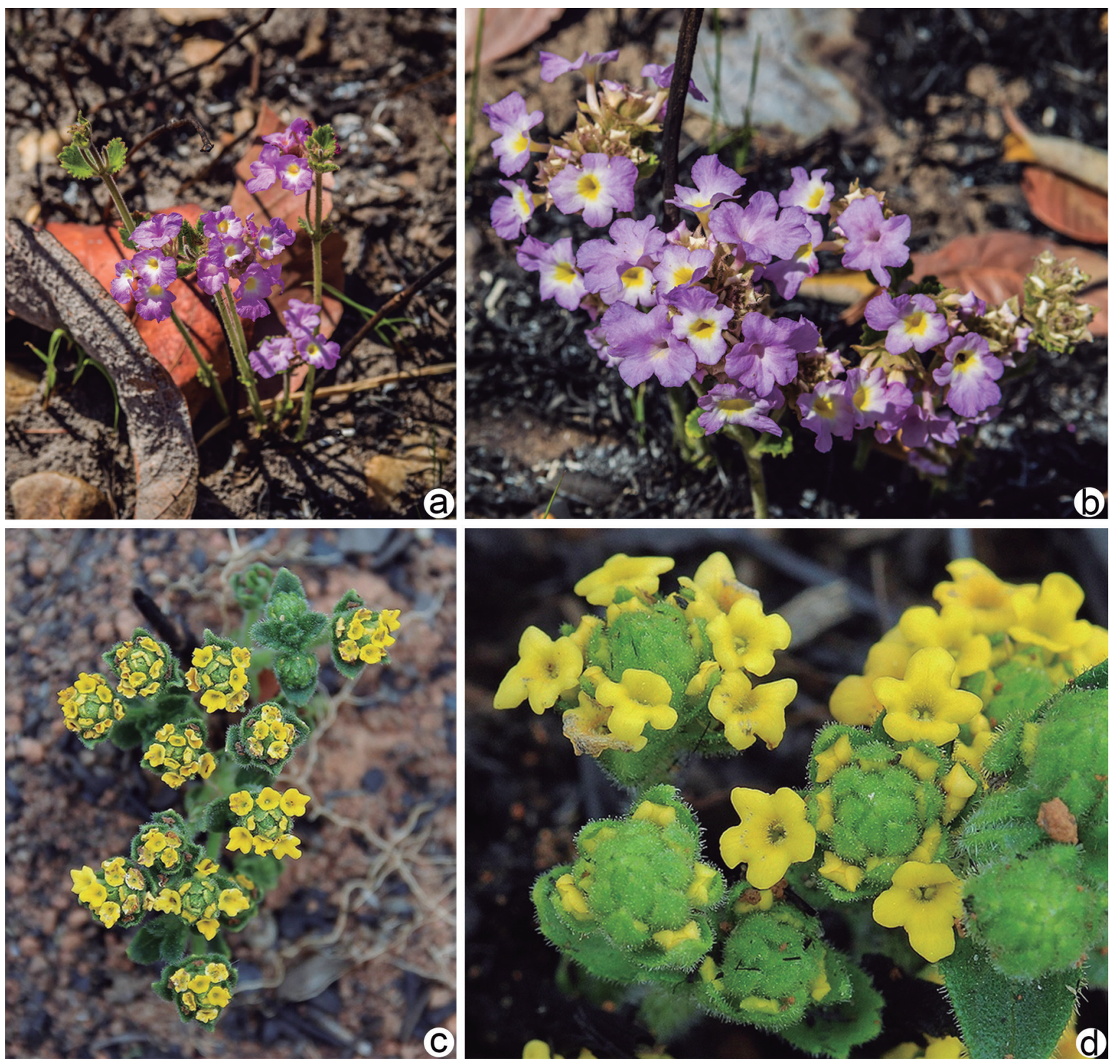

Figura 1. Espécies de Verbenaceae restabelecidas. a-b. Lantana glaziovii. c-d. Lippia nana. (Fotografias a-b: Suzana Martins; c-d: Mauricio Mercadante).

Figure 1. Reestablished Verbenaceae species. a-b. Lantana glaziovii. c-d. Lippia nana. (Photos a-b: Suzana Martins; c-d: Mauricio Mercadante). 
nas coleções de herbário. Lantana glaziovii foi coletada com flores nos meses de janeiro, agosto, setembro, outubro, novembro e dezembro, e com frutos em agosto e outubro. Quando coletados logo após a passagem do fogo, os espécimes atingem aproximadamente até $10 \mathrm{~cm}$ de altura, entretanto, sem a passagem do fogo florescem alcançando até $20 \mathrm{~cm}$ de altura. Devido à ampla distribuição e ocorrência em áreas protegidas, a espécie se enquadra na categoria "Menos Preocupante" (Least Concern, LC) (IUCN 2017).

Material examinado: BRASIL. Distrito FeDERAL: Lago Norte, 30-VII-1983, R.C.F. de Carvalho 123 (BHCB, CESJ); Reserva Ecológica do IBGE, 14-VIII-2000, $M$. Aparecida da Silva 4404 (SPF); Reserva Ecológica do IBGE, 3-X-2002, M. Aparecida da Silva 5397 (CESJ, IBGE). GoIÁs: 1894-95, A.F.M. Glaziou 21892 (BR, G, NY, P, SI); Alto Paraíso de Goiás, 30-I-2012, J.F.B. Pastore \& H. Moreira 4069 (CEN); Serra do Caiapó, 21-VIII-1964, H.S. Irwin \& T.R. Soderstrom 7143 (RB). Mato Grosso: Serra das Araras, Alto Paraguai, 23-X1995, B. Dubs 1886 (MBM, NY); Rio Paraguai, V-1927, Smith 212 (K). Minas GeraIs: Diamantina, 3-XI-1937, M. Barreto 9416 (NY); Serro, próximo a divisa com Presidente Kubitscheck 30-X-2007, F.R.G. Salimena et al. 2540 (CESJ). São PaUlo: Pedregulho, Parque Estadual das Furnas do Bom Jesus, 31-VIII-2003, D. Sasaki \& A.F. Sartori 763 (K, SPF); São Carlos, 17-IX1938, J.E. Rombouts 2680 (NY). Tocantins: Paranã, 23X-2003, G. Pereira-Silva \& G.A. Moreira 11024 (CEN).

2. Lippia nana Schauer, Prodr. 11: 582. 1847. Figura $1 \mathrm{c}-\mathrm{d}$

Tipo-Brasil. GoIÁs: Serra do Urubu, J.B.E. Pohl (Lectótipo (aqui designado) W (W 0074834); isolectótipos W (W0074835), (K000470909)).

Subarbustos 5-10 cm alt., dioicos, ramos cilíndricos, viscosos, pubescentes, internós $0,2-1,5 \mathrm{~cm}$ compr., sistema subterrâneo desenvolvido. Folhas opostas, sésseis; lâminas 1,5-9 × 0,5-4 cm, cartáceas, oblanceoladas a obovadas, base longo-atenuada, ápice agudo, margem inteira até a porção média, crenada em direção ao ápice, face adaxial e abaxial viscosas, estrigosas. Inflorescências 4-8 × 6-9 mm, hemisféricas, pedúnculo $2-5 \mathrm{~cm}$ compr., viscoso; brácteas 3-5 mm compr., dispostas espiraladamente, membranáceas, verdes, viscosas, estrigosas; cálice ca. $2 \mathrm{~mm}$ compr., tubuloso, 4-dentado, verde, viscoso, pubescente; corola ca. $5 \mathrm{~mm}$ compr., zigomorfa, amarela, pubescente. Flores estaminadas: brácteas lanceoladas, estames inseridos na metade do tubo da corola, pistilódio ca. 0,5 mm compr. Flores 
pistiladas: brácteas ovadas, ovário ca. 1,5 mm compr., 2-carpelar, 2-locular, lóculos 1-ovulados. Fruto do tipo esquizocarpo formado por 2 mericarpos, ca. $3 \mathrm{~mm}$ compr., superfície externa lisa.

Lippia seção Dioicolippia Tronc. reúne plantas dioicas, com xilopódio desenvolvido e flores amarelas, dimorfas, dispostas em espigas capituliformes axilares (Romero 2000). Lippia nana é um subarbusto que apresenta todas estas características, e indumento viscoso distribuído por toda a planta, caráter compartilhado com Lippia pumila, tendo sido base para sua sinonimização (Salimena \& Múlgura 2015). É importante destacar que o nome Lippia nana não foi incluído na revisão da seção Dioicolippia (Romero 2000), tratando-se, portanto, de um táxon negligenciado. Com base na análise morfológica das coleções-tipo e de outras coleções de Lippia foi possível reconhecer Lippia nana como uma espécie distinta, sendo aqui restabelecida.

No protólogo, Schauer (1847) cita o espécime coletado por J.B.E. Pohl, proveniente da Serra do Urubu, depositado em W (h. caes. vindob.). Duas exsicatas de Lippia nana foram encontradas no respectivo herbário: W0074834 e W0074835. Portanto, um lectótipo deve ser designado (Turland et al. 2018). Após um exame cuidadoso das coleções, o espécime W0074834 é selecionado como lectótipo, uma vez que é o mais abundante, mostra todas as características morfológicas descritas no protólogo e encontra-se em excelente estado de conservação.

As características diagnósticas que permitem diferenciar Lippia nana de Lippia pumila estão relacionadas principalmente ao hábito e à forma das folhas. Lippia nana é um subarbusto de até $10 \mathrm{~cm}$ de altura, com internós frequentemente inconspícuos, folhas oblanceoladas a obovadas, com base longoatenuada e margem crenada na porção superior. Já Lippia pumila é um subarbusto de $20-30 \mathrm{~cm}$ de altura com internós conspícuos de $2-6 \mathrm{~cm}$ de comprimento, folhas ovado-elípticas, base cuneada a obtusa e margem serreada a partir da porção média (tabela 2 ).

As espécies também apresentam distribuição geográfica distinta, enquanto Lippia nana é endêmica dos cerrados e campos rupestres do Distrito Federal, Goiás e Minas Gerais, Lippia pumila corre nos campos com afloramentos rochosos do Paraná e Rio Grande do Sul. Lippia nana foi coletada com flores nos meses de agosto a dezembro e com frutos em outubro e novembro. Apresenta EOO de 115.216,795 $\mathrm{Km}^{2} \mathrm{e}$ AOO de $20 \mathrm{Km}^{2}$, sendo enquadrada na categoria "Em

Tabela 2. Comparação entre os caracteres morfológicos de Lippia nana e Lippia pumila.

Table 2. Comparison between the morphological characters of Lippia nana and Lippia pumila.

\begin{tabular}{lcc}
\hline Caracteres & Lippia nana & Lippia pumila \\
\hline Altura da planta & $5-10 \mathrm{~cm}$ & $20-30 \mathrm{~cm}$ \\
Comprimento dos internós & $0,2-1,5 \mathrm{~cm}$ & $2-6 \mathrm{~cm}$ \\
Forma das folhas & oblanceoladas a obovadas & ovado-elípticas \\
Base da lâmina foliar & longo-atenuada & cuneada a obtusa \\
Margem da lâmina foliar & crenada na porção superior & serreada a partir da porção média \\
\hline
\end{tabular}

Perigo" (Endangered, EN) (IUCN 2017). Sua área de ocorrência está impactada devido à expansão de atividades agrícola e pecuária, queimadas e invasão de espécies exóticas que ocasionam o declínio na qualidade do hábitat (Salimena et al. 2013).

Material examinado: Distrito Federal: Poço Azul, 30-X-2003, J.F.B. Pastore et al. 719 (CEN); Reserva Ecológica do IBGE, 13-IX-2000, M. Aparecida da Silva 4500 (IBGE). Goiás: Serra do Urubu, J.B.E. Pohl (K, W); Aparecida de Goiânia, 1-XII-2002, J.F.B. Pastore \& E. Suganuma 131 (CEN); Jataí, a $20 \mathrm{Km}$ do Ribeirão Ariranha, 20-IX-1973, J.A. Rizzo 9301 (UFG). Minas Gerais: estrada São Roque de
Minas para Sacramento, $5 \mathrm{~km}$ da sede, 16-X-1994, R. Romero et al. 1267 (CESJ, HUFU); próximo à garagem de pedras, 18-X-1994, R. Romero et al. 1337 (CESJ, HUFU); 18-X-1994, R. Romero et al. 1363 (HUFU); Sacramento, 21-VIII-1994, J.N. Nakajima et al. 433 (CESJ, HUFU).

\section{Agradecimentos}

Os autores agradecem ao Dr. Luiz Menini Neto, Dr. Vinícius Antônio de Oliveira Dittrich e aos revisores anônimos pela revisão do manuscrito. À Coordenação de Aperfeiçoamento de Pessoal de Nível Superior (CAPES), pela concessão de bolsa de mestrado ao primeiro autor. 


\section{Literatura citada}

Atkins, S. 2004. Verbenaceae. In: K. Kubtzki \& J.W. Kadereit (eds.). The Families and Genera of Vascular Plants. Springer-Verlag. v. 7, pp. 449-468.

Bachman, S., J. Moat, A. W. Hill, J. de la Torre, \& B. Scott. 2011. Supporting red list threat assessments with GeoCAT: Geospatial conservation assessment tool. ZooKeys 150: 117-126.

Bentham, G. 1876. Verbenaceae. In: G. Bentham \& J.D. Hooker (eds.). Genera Plantarum. Reeve and Co., London, v. 2, pp. 1131-1160.

BFG (The Brazil Flora Group). 2015. Growing knowledge: an overview of seed plant diversity in Brazil. Rodriguésia 66: 1085-111.

Briquet, J. 1895. Verbenaceae. In: A. Engler \& K. Prantl (eds.). Die natürlichen Pflanzenfamilien IV, 3a. W. Engelmann, Leipzig, pp. 132-182.

Gonçalves, E.G. \& Lorenzi, H. 2007. Morfologia vegetal: organografia e dicionário ilustrado de morfologia das plantas vasculares. Instituto Plantarum, Nova Odessa.

Harris, J.G. \& Harris, M.W. 2003. Plant identification terminology: an illustrated glossary. 2ed. Spring Lake Publ., Spring Lake.

IUCN (International Union for Conservation of Nature). 2017. Guidelines for Using the IUCN Red List Categories and Criteria. Version 13. Disponível em http:// www.iucnredlist.org/documents/RedListGuidelines.pdf (acesso em 22-XI-2018).

Junell, S. 1934. Zur Gynäceummorphologie und Systematik der Verbenaceen und Labiaten. Symb. Bot. Upsal 4: 1-219.

Lu-Irving, P. \& Olmstead, R.G. 2012. Investigating the evolution of Lantaneae (Verbenaceae) using multiple loci. Botanical Journal of the Linnean Society 171: 103-119.

Moldenke, H.N. 1940. Notes on new and Noteworthy. Phytologia 1: 462-466.
Moldenke, H.N. 1965. Materials toward a monograph of the genus Lippia I. Phytologia 12: 174.

Radford, A.E., Dickinson, W.C., Massey, J.R. \& Bell, C.R. 1974. Vascular Plant Systematics. Harper Collins, New York.

Romero, M.E.M. 2000. Las especies de Lippia L. sect. Dioicolippia Tronc. (Verbenaceae). Candollea 55: 227-254.

Salimena, F.R.G., Kutschenko, D.C., Monteiro, N.P. \& Mynssen, C. 2013. Verbenaceae. In: G. Martinelli \& M.A. Moraes (eds.). Livro vermelho da Flora do Brasil. CNCFlora, Rio de Janeiro, pp. 1010-1016.

Salimena, F.R.G. \& Múlgura, M.E.M. 2015. Notas taxonômicas em Verbenaceae do Brasil. Rodriguésia 66: 191-197.

Silva, T.R.S. 1999. Redelimitação e revisão do gênero Lantana L. (Verbenaceae) no Brasil. Universidade de São Paulo, São Paulo.

Silva, T.R.S. \& Salimena, F.R.G. 2002. Novas combinações e novos sinônimos em Lippia e Lantana (Verbenaceae). Darwiniana 40: 57-59.

Thiers, B. 2018. Index Herbariorum: A global directory of public herbaria and associated staff. New York Botanical Garden's Virtual Herbarium. Disponível em http://sweetgum.nybg.org/ih/ (acesso em 28-XII-2018).

Troncoso, N.S. 1974. Los Géneros de Verbenáceas de Sudamérica extratropical. Darwiniana 18: 295-412.

Turland, N.J., Wiersema, J.H., Barrie, F.R., Greuter, W., Hawksworth, D.L., Herendeen, P.S., Knapp, S., Kusber, W.-H., Li, D.-Z., Marhold, K., May, T.W., McNeill, J., Monro, A.M., Prado, J., Price, M.J. \& Smith, G.F. 2018. International Code of Nomenclature for algae, fungi, and plants (Shenzhen Code). Koeltz Botanical Books, Glashütten, Regnum Vegetabile. 